\title{
Minimizing Information Asymmetry Interference using Optimal Channel Assignment Strategy in Wireless Mesh Networks
}

\author{
Gohar Rahman ${ }^{1}$, Chuah Chai Wen ${ }^{2}$ \\ Faculty of computer science \& information Technology \\ Universiti of Tun Hussein Onn Malaysia, 86400, Johor Malaysia
}

\author{
Sadiq Shah ${ }^{3}$ \\ Department of Computer Science \\ FATA University, FR Kohat, Pakistan
}

\author{
Misbah Daud ${ }^{4}$ \\ Institute of Business and Management Sciences \\ The University of Agriculture, Peshawar, Pakistan
}

\begin{abstract}
Multi-radio multi-channel wireless mesh networks (MRMC-WMNs) in recent years are considered as the prioritized choice for users due to its low cost and reliability. MRMCWMNs is recently been deployed widely across the world but still these kinds of networks face interference problems among WMN links. One of the well-known interference issue is information asymmetry (IA). In case of information asymmetry interference the source mesh nodes of different mesh links cannot sense each other before transmitting data on the same frequency channel. This non-coordination leads to data collision and packet loss of data flow and hence degrades the network capacity. To maximize the MRMC-WMN capacity and minimize IA interference, various schemes for optimal channel assignment have been proposed already. In this research a novel and near-optimal channel assignment model called Information Asymmetry Minimization (IAM) model is proposed based on integer linear programming. The proposed IAM model optimally assigns orthogonal or non-overlapping channels from IEEE 802.11b technology to various MRMC-WMN links. Through extensive simulations we show that our proposed model gives $28.31 \%$ network aggregate network capacity improvement over the existing channel assignment model.
\end{abstract}

Keywords-Wireless mesh network; information asymmetry interference; channel assignment; integer linear programming; coordinated interference

\section{INTRODUCTION}

In recent years wireless mesh network (WMN) has become a better option for users as it is reliable, self-configurable and low cost technology. In wireless mesh network (WMN) three types of nodes are involved used for communication i.e. mesh routers, mesh clients and gateway nodes [1]. Mesh routers forms a mesh topology and are connected with each other. These routers forwards packets on behalf of the other nodes called mesh clients as mesh clients may not be within each other's direct wireless transmission range.

Wireless mesh network are almost static or minimal mobile that makes a backbone network called mesh backbone. PDAs, desktop systems, laptops, smart phones etc. are traditional mesh client nodes. All these clients access mesh routers to communicate and with each other and with outside world using gateways nodes [2, 3]. Each node in the WMN gives the end users a reliable environment due to its multiple path and redundant links. In case of failure on single route the flow of data is sent on the alternate redundant path. That is the reason why WMNs are reliable and self-configurable. Wireless mesh network has static mesh routers or have minimum mobility.

The complete architecture of MRMC-WMN is shown in Fig. 1. Wireless mesh network can have single or multiple radios. In our research we have taken multi-radios architecture where the mesh nodes are equipped with multiple radios. These multiple radios perform significant role in maximizing the aggregate network capacity. Recently researches have adopted the use of multiple interfaces concept. Further multiple channels at MAC layer can be assigned at the same time to a wireless mesh node to take advantage by using multiple channels. Compared to single radio architecture multiple radios enhance the overall network capacity.

\section{A. Multi Radio WMNs}

In a single-radio WMN each mesh node operates on only one frequency channel at single time slot. Generally single radio in wireless mesh network are considered the weakest approach to form a mesh network. To overcome the single radio issue another alternative approach has been developed that is called multi-radio wireless mesh network. In case of MRMC-WMN each mesh router is equipped with multiple interfaces or radios. Due to the presence of multiple interfaces on each mesh router multiple channels form IEEE $802.11 \mathrm{~b}$ can be assigned to mesh node. This strategy improves the network throughput up to a great extent [4]. In case of single-radiosingle channel (SRSC) design each mesh node has only one radio and that radio can use only one frequency channel. The main drawback of SRSC structural design is less throughput and limited capacity due to limited number of channels [5]. Keeping in view the disadvantages of single-radio design the alternative design is multi-radio-multi-channel (MRMC) structure. Further multiple channels can be assigned to each node. The multi-radio multi-channel leads to simultaneous 
communication among mesh nodes and hence network throughput can be maximized by using optimal channel assignment strategies.

\section{B. IEEE 802.11b and WMNs}

The wireless technology used in this study is $802.11 \mathrm{~b}$, which operates in the $2.4 \mathrm{GHz}$ band. The IEEE $802.11 \mathrm{~b}$ standard consists of 11 channels, three of which do not overlap, namely 1, 6, and 11. Each channel has a transmission capacity of $11 \mathrm{mbps}$. Nodes can only communicate within the transmission range of each other. Due to the limited number of available channels, nodes may interfere with each other for channel access. Early research has classified these issues, namely information asymmetry interference, near hidden and far hidden interference [6]. In this research we are going to minimize information asymmetry problem so as to maximize WMN network capacity. In order to overcome the problem of information asymmetry, various optimization and channel allocation models have been proposed. The main purpose of the previous channel allocation mechanism is to reduce channel interference between different wireless links. The focus of this study is to minimize both coordinated and information asymmetry interference schemes.

\section{Coordinated Interference}

Coordinated interference is the case where the transmitters of different links are each other's carrier sensing range. Fig. 2 represents coordinated interference along with transmission range and carrier-sensing range of the source node al of link $\mathrm{L} 1$ respectively. Both the link L1 and L2 are coordinated as they are sharing the same frequency channel 1. For coordinated interference CSMA/CA protocol is used to share the channel capacity among links. CSMA / CA is a technique in which a wireless node senses a frequency channel before transmitting data. It checks the media for transmission before sending it.

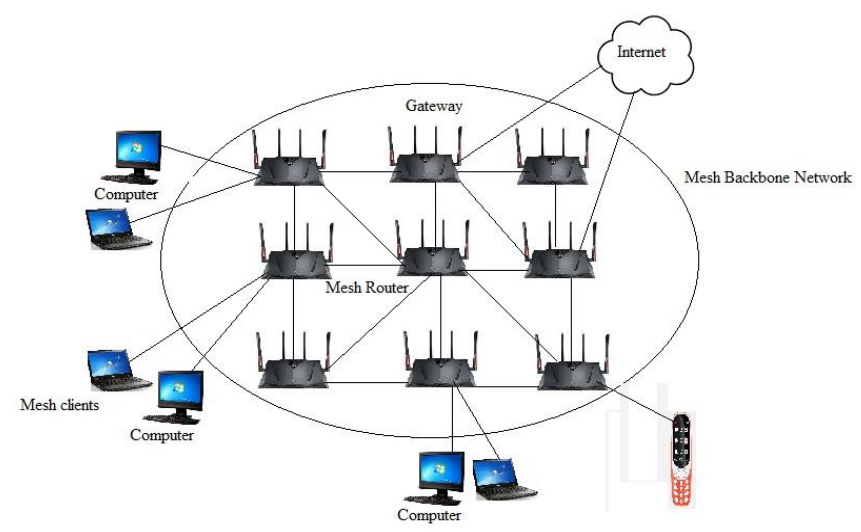

Fig. 1. Architecture of Wireless Mesh.

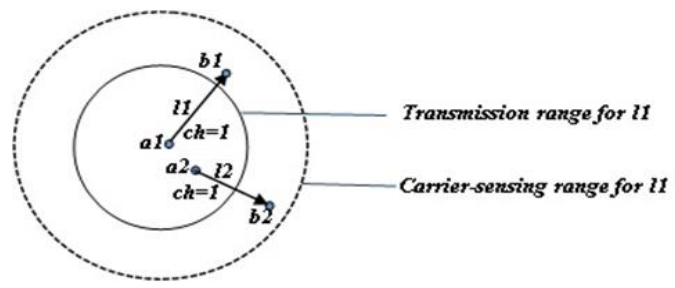

Fig. 2. Coordinated Relationship between Links L1 and L2.
If the transmission medium is found idle then the node transmits data. If the medium is sensed busy then the sender node starts waiting for a long period of time in order to access a frequency channel. It may happen that two or more nodes sense an idle channel and starts sending their flows at the same time, this can cause collision among their data flows. This kind of problem is solved by handshake mechanism which is used by CSMA/CA also known as Request-ToSend/Clear-To-Send technique. In case of RTS/CTS mechanism the station sends a RTS packet in order to gain medium for its data transmission. After RTS if the medium is found free, the receiving station on the receiving side responds back with a Clear-To-Send (CTS) signal. The sending then starts transmitting data after it sees the CTS signal. Earlier studies done so far shows that coordinated interference (CO) is not harmful as the sending nodes shares a frequency channel among multiple nodes. Apart from CO interference another well-known interference type is information asymmetry that is discussed in the next Section 1.4.

\section{Information Asymmetry Interference}

In information asymmetry interference the source nodes of any two are outside the carrier sensing ranges of each other. For example in Fig. 3 we have three links $(s 1, d 1),(s 2, d 2)$ and $(s 3, d 3)$ which are operating on the same IEEE $802.11 \mathrm{~b}$ frequency channel. The following condition in Eq. (1) and Eq. (2) creates the information asymmetry interference between $(s 1, d 1),(s 2, d 2)$ and between $(s 1, d 1),(s 3, d 3)$.

$d(s 2, d 1)<C R$

$d(s 3, d 1)<C R$

Here $d$ denotes the geographic distance between WMN nodes. Similarly $C R$ represents the interference range or carrier-sensing of each mesh node. Source nodes $s 2$ and $s 3$ are in the CR of receiver node $d l$. In this case if all the given links in Fig. 3 are assigned the same frequency channel then the flow on link $(s 1, d 1)$ may interfere. In Fig. 2 the solid line circle shows the $C R$ of the source node $s l$ while the dotted circle represents carrier-sensing range of the receiving node $d 1$.

Moreover studies have shown that information asymmetry interference till now is not solved and handled carefully by the well-known CSMA/CA (carrier sense multiple access) protocol. CSMA/CA protocol functions inside the $\mathrm{CR}$ ranges while information asymmetry (IA) problems arises external the CR of sending nodes. The main purpose of this research is to propose a linear programming model to minimize the information asymmetry problem and to maximize the WMN network capacity. In the next section, we present a detailed survey on Interference issue in WMN.

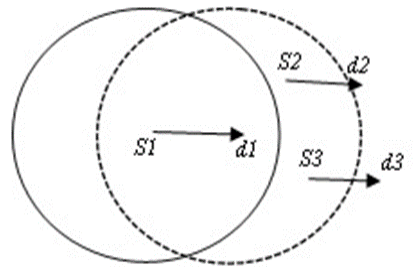

Fig. 3. Information Asymmetry Links in WMN. 


\section{RELATED WORK}

Reena et al. [7] identified an issue in multi-radio wireless mesh network that was local link failure problem. They presented a system named as autogenesis network reconfiguration system (ARS), the system ensured the protection of local link failure in WMN and also maintain the network throughput. They discussed some of the changes that may be encountered through ARS, such as changes in local radio and channel assignments. The results for this scheme have been taken from Ns 2 simulator and it was concluded that autogenesis network reconfiguration system gives better result over other previous models and the channel efficiency increased up to $90 \%$.

Valarmozhi et al. [8] discussed issues of wireless mesh network were studied. Different issues such as channel assignment, power control and routing problem of wireless mesh network were discussed. It was analysed that nodes armed with numerous radios and operating on several channels can cause reduction in interference issue and can improve the capacity of a network. The author stated that one of the research areas is the mechanism of topology control. Ding et al. [9] focused on multi hop wireless mesh network, each router in a network has multiple radios and for the purpose of communication multiple channels are available. The frequency channels were assigned to different interfaces to the WMN to minimize the overall interference. The author formulated a channel assignment scheme that was an NP-hard problem.

Chaudhry et al. [10] evaluated that MRMC-WMNs is a promising technology, each node is equipped with multiple radios and each radio is assigned a single channel. They discussed certain issues like power control, optimal channel assignment problem and routing process in MR-MC WMNs. The author presented a novel topology control scheme and formulated different NP hard complexity problems in MR-MC WMNs. It was concluded to solve these stated types of issues in MR-MC WMNs that could supply more favourable services to end user.

Hoque et al. [11] says that existing channel assignment schemes were discussed together with the significance of efficiently channel allocation to various nodes in the network. They analysed that channel assignment performs great role to determine the network overall performance. The author further divided the interfering links into two main parts that is coordinated interference (CO) and non-coordinated (nCO) interference. A new clique-based clustering channel assignment scheme was presented for the minimization of $\mathrm{CO}$ interference and nCO interference. The network is divided into different clusters and channel assignment was done on the basis of proposed algorithm. The main objective was to decrease $\mathrm{CO}$ and $\mathrm{nCO}$ interference in the network. Through various simulations the result showed that the CCCA proposed scheme can reduce the end to end delay and can maximize the network capacity of wireless mesh network. Sani and Kumar [12] proposed a problem of channel interference in MRMCWMN. They identified an efficient dynamic cross layer design named as R-CA. Through simulation results it was concluded that the network throughput enhanced by this proposed channel assignment algorithm.

Further, Makram et al. [13] explained different issues of MRMC-WMN and various solution and approaches were presented. They worked on partially overlapping channel assignment and on different issues and challenges related to MRMC-WMN that is routing issues in designing of wireless mesh network. On the basis of these schemes different analysis are made and existing mechanism are classified. Subramanian et al. [14] has been investigated that the reduction in capacity of multi-radio WNN due to the interference between links in wireless communication. They worked on cluster channel assignment mechanism and formulated problems occurred due to channel assignment among different nodes in wireless mesh networks. This approach was used for the purpose of minimization of link interference of various communication links and improving of network aggregate capacity. This method has two main portions. The first portion consists clustering of WMN to overcome the local problem within the cluster. Secondly, to make use of frequency channel efficiently within the cluster. A cluster-based channel allocation mechanism is employed to minimize the complexity of channel allocation and to reuse channels in different clusters. Finally, the results of sparse and dense networks are shown.

Yin et al. [15] presented the issue of joint channel assignment and routing issue. The author stated that the problem is NP-complete. The first phase they use a model called rate-variable model that enhances the network aggregate throughput. Second, they proposed a mathematical programming model. The model formulates the channel assignment and routing problem by deriving an integer linear programming (ILP) problem. Their simulation and experimental results show that their proposed approach effectively increases the network throughput. Cooper et al. [16] divided the channel interference into $\mathrm{nCO}$ and $\mathrm{CO}$ interference. The NCO interference is further divided into near-hidden, information asymmetry and far-hidden interfering links. It is also proposed that the special effects of these interfering inks in MRMC-WMN and determined the total possibilities of packets losses. They also proposed a model called an analytical model for minimizing $\mathrm{CO}$ interference and also nCO interference. It was concluded in the end that the nCO interference is much destructive than the CO Interference among mesh links. Channels for network throughputs at various node degree constraints as compared to simpler interference model.

Shah et al. [17] worked on an issue in MRMC-WMNs that is link interference problem for both coordinated and noncoordinated interference. The author proposed a linear algebraic model which is entirely based on non-coordinated interference. The comparison of model results for both sparse and dense topologies was done through OPNET simulator. From the results they concluded that the proposed model gives considerable network capacity improvement of sparse network over the dense network i.e. $19 \%$. 


\section{PROPOSED MODEL FORMULATION}

In this section, we are formulating a linear programming model to minimize information asymmetry interference. The proposed model is termed as information asymmetry minimization (IAM) model. The IAM model contains of a binary decision variable, one objective function and a set of constraints.

\section{A. Proposed IAM Model}

The proposed IAM model is given below where the detailed description of all the parts of the proposed is given in detail.

1) Binary decision variable: the function of binary decision variable is to assign IEEE $802.11 \mathrm{~b}$ channel $\mathrm{j}$ to a link $i$. In case of successful channel assignment the value is 1 otherwise 0 . If the value is 1 then the decision variable states that the directed link $i$ is transmitting data on channel $j$ otherwise it is considered 0 . Our binary decision variable is represented through Eq. (3).

$x_{i, j}=\left\{\begin{array}{ll}1 & \text { if a directed link } i \text { operates on channel } j \\ 0 & \text { otherwise }\end{array}\right\}$

\section{B. Objective Function}

The main objective of the proposed IAM model is the maximization of the MRMC-WMN capacity. The given objective function in Eq. (4) adds all the WMN edges $E$ with flow $f$ over the link $i$ fulfilled after optimal channel assignment scheme. Here $\lambda i$ is fraction of flow successfully transmitted on link $i$.

$\max \sum_{i \in E} \sum_{j \in H} x_{i, j} \cdot \lambda_{i} \cdot f_{i}$

Here $H$ is the set of IEEE 802.11b non-overlapping channels i.e. 1, 6, 11 and $E$ is the set of mesh edges (links).

\section{Constraints Set}

Along with objective function there are some constraints in that represent the restrictions on the optimization model. The proposed constraints for our proposed model are represented through Eq. (5), Eq. (6) and Eq. (7).

1) Single channel constraint: Single channel constraints ensure that only one channel is assigned a single link. This means that each link in set E must be rotated on only one channel. A single channel for each link constraint explicitly indicates that if $\mathrm{i}$ is a link, then the set $\mathrm{H}$ is assigned to the group on only one channel $\mathrm{j}$.

$\sum_{j \in H} x_{i, j}=1 \quad \because \forall i \in E, j \in H$

2) Coordinated interference constraint: Coordinated links as mentioned earlier do not create severe interference instead they share the capacity of the frequency channel. The frequency channel capacity is divided among those links that are coordinated with each other. The constraint is already proposed by [17] and is represented in Eq. (6). Here $\lambda$ is the fraction of traffic flow fulfilled on link $i$ and $k$ is the coordinated links set of link $i$.

$$
\begin{aligned}
& x\left(e_{i}, c_{j}\right) \cdot \lambda\left(e_{i}\right) \cdot f\left(e_{i}\right)+\sum e_{k} \in N c o\left(e_{i}\right)^{x\left(e_{k}, c_{j}\right) \cdot \lambda\left(e_{k}\right) \cdot f\left(e_{k}\right) \leq C c_{j}} \\
& \forall e_{i} \in H, \forall c_{j} \in P
\end{aligned}
$$

3) Information Asymmetry Minimization Constraint: This is the proposed constraint of this research that is merged with the existing model to minimize the information asymmetry interference existing. The channel assignment strategy restricts that two IA links will not operate on common or fully overlapping frequency channel. Here $I A(i)$ is the set of information asymmetry links of link $i$. Only one channel $j$ can be assigned to either $i$ or $k$ for IA set.

$$
x_{i, j}+\sum_{k \in I A(i)} x_{k, j} \leq 1 \quad \forall i, k \in E, \forall_{j} \in H
$$

\section{RESULTS AND DISCUSSION}

This section is divided into three sections. In the first part, we created multiple MRMC-WMN topologies in MATLAB shown in Fig 4. The purpose of this topology construction is the identification of information asymmetry and coordinated links of each link in the network. We have created four different MATLAB topologies. The identified results are then given to A Mathematical programming language (AMPL) tool to get near optimal channel assignment results from the propose IAM model. Further the channel assignment results is given to OPNET. In the next section extensive OPNET simulations have been done to verify the model results. Results are discussed in detail along with each OPNET result.

\section{A. Multi-Radio Topology Construction}

Fig. 4, four WMN topologies has been created in MATLAB. These topologies consists of 10, 15, 20, 25 nodes respectively. During experimentation the number of nodes is kept varying. The number of nodes is increased from 10 nodes to 25 nodes. In the end all the results taken from this scalable nature is averages. The interference effect of IA is checked on topologies with increasing number of nodes. We have assumed that the each mesh node has a transmission range (Tr) is thirty (30) meters while the carrier-sensing range (CR) also called interference rang is 78 meters that is 2.6 times of transmission range (Tr). From all the four topologies coordinated and IA links are identified. In Table 2 all the coordinated and IA interfering links of each link is given. For example link $(13,14)$ is considered from Fig. 4(c) are the set of coordinated links i.e. $(2,3)(4,5)(3,4)(11,12)(7,8)(12,13)$ $(13,14)(16,17)(14,15)(17,18)(18,19)(19,20)(21,22)(23,24)(22$, 23) while the set of given information asymmetry (IA) mesh edges is $(24,25)$. Apart from this we have identified such kind of interference for all the remaining MRMC-WMN topologies. The purpose behind this topology is the identification of those links that are Information asymmetry links. The results taken from these WMN topologies are then given to AMPL (A Mathematical Programming Language) tool in order to get the optimal channel assignment scheme. In Fig. 4 all the WMN topologies are shown. The simulation parameters for this research are given in Table 1. 
TABLE I. PARAMETER USED DURING SIMULATION RESULTS

\begin{tabular}{|l|l|}
\hline Parameter & Value \\
\hline Number of Mesh Nodes & $10,15,20,25$ \\
\hline Radios per node & 2 \\
\hline Channel Capacity & 11 Mbps (Max.) \\
\hline Transmission Range & 30 meters \\
\hline Carrier-Sensing Range & 78 meters \\
\hline Simulation time & 3 minutes \\
\hline
\end{tabular}

\section{B. Channel Assignment Result of IAM Model}

The channel assignment results obtained from proposed model is near optimal solution. The entire paths among mesh node are taken single link paths. Each link flow demand has been varied from 100 packet per second to 500 packets per second on each source mesh node. In this section IAM model results have been taken in AMPL using Gurobi solver. Although there are multiple solver exists in AMPL that can solve various linear and non-linear problems. But in this paper the model proposed is linear that is the reason why Gurobi was preferred.

In Table 2 all the coordinated and information asymmetry links have been identified for each source link. The total links taken are twenty. For all other topologies given in Fig. 4, we have followed the same approach by identifying information asymmetry and coordinated edges. These links are given as input to AMPL solver that executes the proposed IAM model. The channel assignment results obtained from proposed model is near optimal solution. The entire path among mesh nodes are taken single link path. Each link flow demand has been varied from 100 packet per second to 500 packets per second on each source mesh node In Table 2 all the coordinated and information asymmetry links have been identified for each source link.

In Table 3 the near optimal channel assignment results are given for Fig. 4(b). Where the dashed circle shows carriersensing range $(\mathrm{Cr})$ of the source node (node 3 in (a)) while solid line circle represents the $\mathrm{CR}$ of the given receiver. The channel assignment results given by IAM model are simulated in OPNET for further verification. A total of four scenarios with multiple mesh network sizes are presented. The objective is to compare the IAM model results with that of the existing channel assignment model.

TABLE II. OPTIMAL CHANNEl ASSIGNMENT RESUlT

\begin{tabular}{|l|l|}
\hline WMN Link & Assigned IEEE 802.11b channel \\
\hline$(1,2)$ & 1 \\
\hline$(2,3)$ & 1 \\
\hline$(3,4)$ & 11 \\
\hline$(4,5)$ & 6 \\
\hline$(5,6)$ & 6 \\
\hline$(6,7)$ & 11 \\
\hline$(7,8)$ & 6 \\
\hline$(8,9)$ & 11 \\
\hline$(9,10)$ & 6 \\
\hline$(10,11)$ & 1 \\
\hline$(11,12)$ & 1 \\
\hline$(12,13)$ & 11 \\
\hline$(13,14)$ & 1 \\
\hline$(14,15)$ & 6 \\
\hline
\end{tabular}

TABLE III. COORDINATED AND INFORMATION ASYMMETRY SET OF ALL LINKS IN FIG.4 (D)

\begin{tabular}{|c|c|c|}
\hline Link & Coordinated Links(CO) & Information Asymmetry Links \\
\hline$(1,2)$ & $(1,2)(6,7)(2,3)(12,13)(11,12)(17,18)(16,17)$ & $(3,4)(7,8)(13,14)(18,19)$ \\
\hline$(2,3)$ & $(1,2)(3,4)(6,7)(7,8)(11,12)(12,13)(13,14)(16,17)(17,18)(18,19)$ & $(4,5)(8,9)(14,15)(19,20)$ \\
\hline$(3,4)$ & $(1,2)(2,3)(4,5)(6,7)(7,8)(8,9)(11,12)(12,13)(13,14)(14,15)(16,17)(17,18)$ & $(9,10)(22,23)$ \\
\hline$(4,5)$ & $(18,19)(19,20)$ & $(24,25)$ \\
\hline$(6,7)$ & $(1,2)(2,3)(3,4)(7,8)(8,9)(11,12)(12,13)(13,14)$ & $(4,5)(9,10)(14,15)(19,20)$ \\
\hline$(7,8)$ & $(2,3)(3,4)(7,8)(8,9)(9,10)(18,19)(19,20)$ & Nil \\
\hline$(8,9)$ & $(2,3)(3,4)(4,5)(6,7)(8,9)(9,10)(12,13)(13,14)(14,15)(19,20)$ & Nil \\
\hline$(9,10)$ & $(2,3)(3,4)(4,5)(6,7)(7,8)(9,10)(13,14)(14,15)(19,20)$ & Nil \\
\hline$(11,12)$ & $(4,5)(7,8)(8,9)(14,15)$ & $(4,5)(14,15)(19,20)(22,23)$ \\
\hline$(12,13)$ & $(1,2)(2,3)(3,4)(12,13)(13,14)(16,17)(17,18)(18,19)(21,22)$ & $(4,5)(14,15)(19,20)(24,25)$ \\
\hline$(13,14)$ & $(2,3)(3,4)(6,7)(11,12)(12,13)(13,14)(14,15)(16,17)(17,18)(18,19)(22,23)$ & $(24,25)$ \\
\hline$(14,15)$ & $(23,24)$ & \\
\hline$(16,17)$ & $(2,3)(3,4)(4,5)(7,8)(11,12)(12,13)(13,14)(14,15)(16,17)(17,18)(18,19)$ & $(9,10)$ \\
\hline$(17,18)$ & $(19,20)(21,22)(22,23)(23,24)$ & $(3,4)(14,15)(19,20)(22,23)$ \\
\hline$(18,19)$ & $(3,4)(4,5)(7,8)(8,9)(12,13)(13,14)(21,22)(22,23)(23,24)(24,25)$ & $(3,4)(13,14)(18,19)(21,22)$ \\
\hline$(19,20)$ & $1,2)(2,3)(3,4)(11,12)(12,13)(13,14)(17,18)(18,19)(21,22)$ & $(4,5)(14,15)(19,20)(24,25)$ \\
\hline$(21,22)$ & $(11,12)(12,13)(13,14)(16,17)$ & Nil \\
\hline$(22,23)$ & $(3,4)(11,12)(12,13)(13,14)(16,17)(17,18)(21,22)(22,23)(23,24)$ & $(14,15)(19,20)(24,25)$ \\
\hline$(23,24)$ & $(3,4)(4,5)(12,13)(13,14)(14,15)(21,22)(22,23)(24,25)$ & $(4,5)$ \\
\hline$(24,25)$ & $(12,13)(13,14)(14,15)(18,19)(22,23)(23,24)$ & $(19,20)$ \\
\hline
\end{tabular}




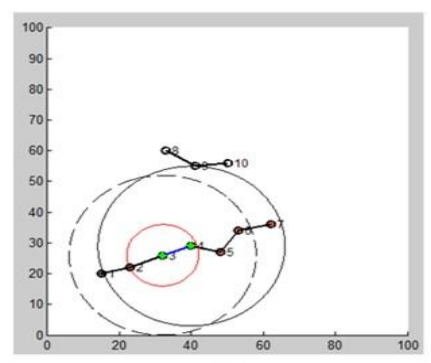

(a)

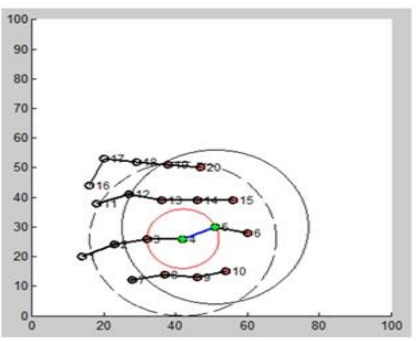

(c)

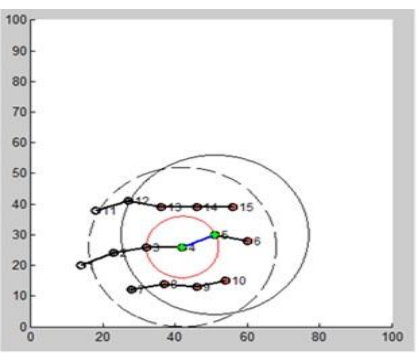

(b)

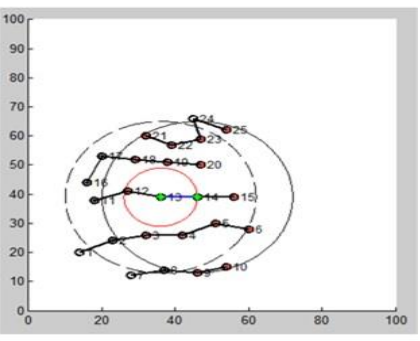

(d)
Fig. 4. (a, b, c, d) Represents WMN to Pologies. Each Consists of 10, 15, 20 and 25 Nodes Respectively.

\section{Simulation Results}

1) Simulation assumptions: In this research, some assumptions taken are given below.

- The channel capacity of each frequency channel is the same.

- Each mesh node is equipped with multiple radios instead of one.

- Each mesh node is kept static and all paths are taken as single link path.

- The carrier-sensing range and transmission power is assumed same for all the mesh nodes.

- Three non-overlapping channels considered are 1, 6 and 11.

Every mesh router or node in the network consist maximum of three radios. Like AMPL results the flow demand, in OPNET the flow demand is kept increasing from 100 packets to 500 packets/sec. For source traffic generation poison traffic generator is used. Table 4 summaries the results of the IAM model results for all the scenarios. The results are taken from AMPL model.

The average improvement of the proposed optimization model shows better capacity improvement over existing model. In the end we have given a percentage analysis that show the improvement of our proposed channel assignment model over existing channel assignment model. Table 4 shows the summary results taken for all the topologies having network size of 10, 15, 20 and 25 node respectively. The flow demand varies from 100 to 500 packets per second. These result show that with increase in the number of nodes the net capacity of the MRMC-WMN increases. Same kind of increase is also occurring with the increase in packet per second from 100 to 500 packets per second.

After simulating all these four topologies in OPNET for the proposed model next we simulate experimented the existing model. The channel assignment result we get from the existing model is tested from the same topologies in Fig. 4. This time we get different result that are given in Table 5. For existing model simulation the parameters kept the same as for the proposed model i.e. flow demand, number of radios, WMN topologies, terrain area etc. For comparative analysis the results of

Proposed IAM and existing model are represented through line charts in Fig. 5. Each chart in Fig. 5 compares result of all the four considered topology i.e. 10, 15, 20 and 25 respectively

Proposed IAM and existing model are represented through line charts in Fig. 5. Each chart in Fig. 5 compares result of all the four considered topology i.e. 10, 15, 20 and 25 respectively

The behaviour of the graph in Fig. 6 shows the overall aggregate capacity increase with the increase in flow demand. The graph clearly shows that the IAM channel assignment model given better capacity results than the existing model. In Table 6 the percentage improvement of IAM model over existing model has been measured for each topology. For this analysis the data has been taken from Tables 4 and 5 .

TABLE IV. IAM MODEL SiMULATION RESUlTS

\begin{tabular}{|l|l|l|l|l|}
\hline $\begin{array}{l}\text { Flow } \\
\text { Demand }\end{array}$ & $\begin{array}{l}\text { Network } \\
\text { Capacity } \\
(\mathbf{1 0 N})\end{array}$ & $\begin{array}{l}\text { Network } \\
\text { Capacity } \\
(\mathbf{1 5 N})\end{array}$ & $\begin{array}{l}\text { Network } \\
\text { capacity } \\
(\mathbf{2 0 N})\end{array}$ & $\begin{array}{l}\text { Network } \\
\text { capacity } \\
(\mathbf{2 5 N})\end{array}$ \\
\hline Packet/sec & Packet/sec & Packet/sec & Packet/sec & Packet/sec \\
\hline 100 & 932.8 & 1403.14 & 1898.09 & 2288.25 \\
\hline 200 & 1866.21 & 2605.88 & 3154.27 & 3195.56 \\
\hline 300 & 2279.24 & 3038.48 & 3599.99 & 3630.54 \\
\hline 400 & 2373.77 & 3160.108 & 3740.64 & 3456.47 \\
\hline 500 & 2447.39 & 3755.65 & 3755.35 & 4155.99 \\
\hline
\end{tabular}

TABLE V. Existing Channel Assignment Model Simulation RESULTS

\begin{tabular}{|l|l|l|l|l|}
\hline $\begin{array}{l}\text { Flow } \\
\text { Demand }\end{array}$ & $\begin{array}{l}\text { Network } \\
\text { Capacity } \\
(\mathbf{1 0 N})\end{array}$ & $\begin{array}{l}\text { Network } \\
\text { Capacity } \\
(\mathbf{1 5 N})\end{array}$ & $\begin{array}{l}\text { Network } \\
\text { capacity } \\
(\mathbf{2 0 N})\end{array}$ & $\begin{array}{l}\text { Network } \\
\text { capacity } \\
(\mathbf{2 5 N})\end{array}$ \\
\hline Packet/sec & Packet/sec & Packet/sec & Packet/sec & Packet/sec \\
\hline 100 & 911.8 & 1371.44 & 1882.08 & 2135.66 \\
\hline 200 & 1544.87 & 2476.59 & 2863.8 & 3033.82 \\
\hline 300 & 1978.04 & 2895.1 & 3290.88 & 3411.26 \\
\hline 400 & 2234.54 & 3130.9 & 3493.34 & 3194.99 \\
\hline 500 & 2310.49 & 3242.91 & 3672.48 & 3238.8 \\
\hline
\end{tabular}




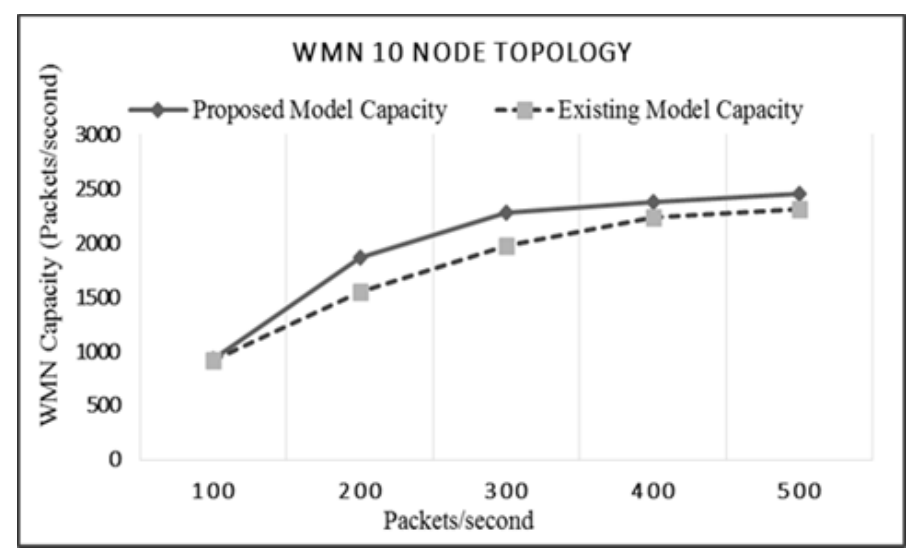

(a)

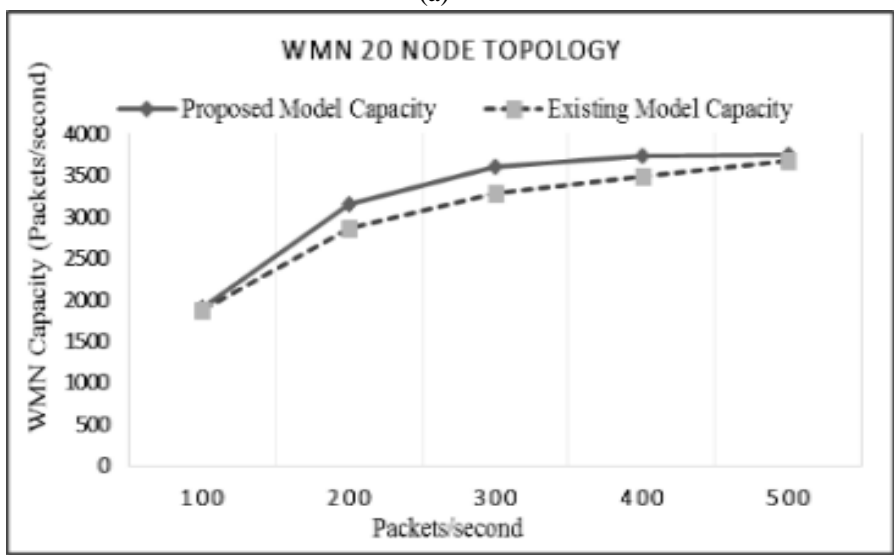

(c)

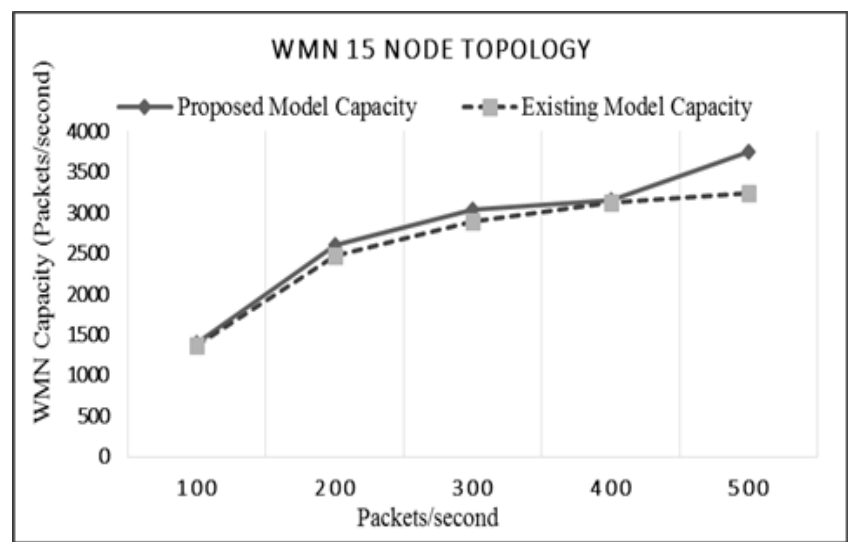

(b)

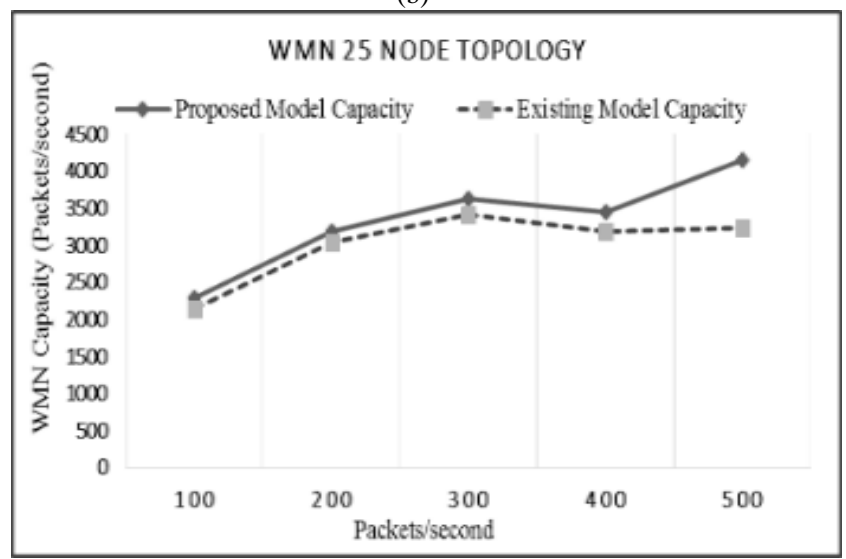

(d)

Fig. 5. Proposed and Existing Model Comparison for (a) 10 Node, (b) 15 Node, (c) 20 Node and (d) 25 Node WMN.

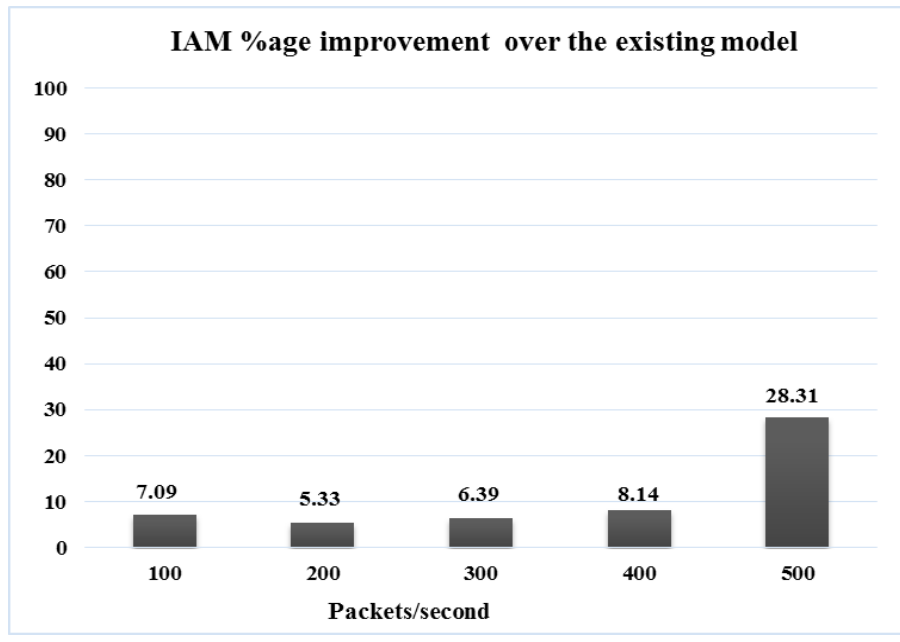

Fig. 6. IAM Percentage Increase over Existing Model Percentage Comparison.

The purpose of this analysis is to show the actual significance and improvement made of our proposed model. It is clear from Table 6 that the percentage improvement is high for those environments where the information asymmetry (IA) interference is high. The maximum percentage increase that IAM model has given over the existing model is $28.31 \%$. This improvement is given for the network topology that has 25 nodes and high data rate i.e. 500 packets.
TABLE VI. Percentage Increase over Existing Model Percentage COMPARISON

\begin{tabular}{|l|l|l|l|l|}
\hline $\begin{array}{l}\text { Flow } \\
\text { Demand }\end{array}$ & $\begin{array}{l}\text { Network } \\
\text { Capacity } \\
(\mathbf{1 0 N})\end{array}$ & $\begin{array}{l}\text { Network } \\
\text { Capacity } \\
(\mathbf{1 5 N})\end{array}$ & $\begin{array}{l}\text { Network } \\
\text { capacity } \\
(\mathbf{2 0 N})\end{array}$ & $\begin{array}{l}\text { Network } \\
\text { capacity } \\
(\mathbf{2 5 N})\end{array}$ \\
\hline Packet/se & $\begin{array}{l}\text { Percentage } \\
\text { Increase }\end{array}$ & $\begin{array}{l}\text { Percentage } \\
\text { Increase }\end{array}$ & $\begin{array}{l}\text { Percentage } \\
\text { Increase }\end{array}$ & $\begin{array}{l}\text { Percentage } \\
\text { Increase }\end{array}$ \\
\hline 100 & 2.30 & 2.24 & 0.90 & 7.09 \\
\hline 200 & 20.73 & 5.17 & 10.14 & 5.33 \\
\hline 300 & 15.17 & 4.88 & 9.36 & 6.39 \\
\hline 400 & 6.23 & 0.93 & 7.07 & 8.14 \\
\hline 500 & 5.92 & 15.68 & 2.17 & 28.31 \\
\hline
\end{tabular}

V. CONCLUSION AND FUTURE WORK

In this research, an optimization model termed as IAM has been presented to perform near-optimal channel assignment. During modelling and experimentation the IAM channel assignment model is compared, regarding network capacity with the existing channel assignment model. Multiple scenarios of 10, 15, 20 and 25 node MRMC-WMN topologies are simulated. It is observed that IAM optimization model performs better results in those environments where the IA interference occurs in high ratio. From the results it is concluded that IAM optimization model provides $28.31 \%$ capacity improvement over existing model. This increase 
occurred in situation where the number of mesh nodes are high i.e. 25 node. These analysis shows that the proposed model performs better in large wireless mesh networks as the chances of information asymmetry interference increases as the size of network grows. In the future the IAM optimization model can be expanded to both orthogonal and partially overlapping channel (POC) assignment. Further, other categories of WMN interference are Near-Hidden and Far hidden node problem.

\section{ACKNOWLEDGMENT}

The work is done with The University of Agriculture Peshawar Pakistan and Tier 1 H082, RMC Universiti Tun Hussain Onn Malaysia. We are thankful to all the stakeholders for helping us throughout this research work.

\section{REFRENCES}

[1] Jin, F. Lv, X. and Liu, X, "Multi-Radio Multi-Channel Protocol for Emergency Wireless Mesh Network", In Proceedings of the 7th International Conference on Computer Engineering and Networks. Shanghai, China, 2017.

[2] Odabasi, S.D, and Zaim, A.H, "A survey on wireless mesh networks, routing metrics and protocols", International Journal of Electronics, Mechanical and Mechatronics Engineering (IJEMME), 2(1), pp. 92104, 2010.

[3] Kareem, T.R. Matthee, K. Chan, H.A. and Ntlatlapa, N, "Adaptive Priority Based Distributed Dynamic Channel Assignment for Multiradio Wireless Mesh Networks", In International Conference on Ad-Hoc Networks and Wireless. Springer, Berlin, Heidelberg, pp.321-332, 2008.

[4] Liu, F. and Bai, Y, "An overview of topology control mechanisms in multi-radio multi-channel wireless mesh networks", EURASIP Journal on Wireless Communications and Networking, 1, pp.324, 2012.

[5] Ren, W. Zhao, Q. Ramanathan, R. Gao, J. Swami, A. BarNoy, A. Johnson, M.P, and Basu, P, "Broadcasting in multi-radio multi-channel wireless networks using simplicial complexes", Wireless Network The Journal of Mobile Computation and Information, 19(6), pp. 1121$1133,2013$.

[6] Garetto, M. Salonidis, T. and Knightly, E.W, "Modeling per-flow throughput and capturing starvation in CSMA multi-hop wireless networks", IEEE/acm transactions on networking (ton), 16(4), pp.864877,2008 .

[7] Reena, J.H. Reddy, K.G. and Srinivas, P.V.S, (2013). "Autogenous Reconfigurable Wireless Mesh Network", Journal of Engineering Science, 2(11), pp.39-49, 2013.

[8] Valarmozhi, A. Subala, M. and Muthu, V, " Survey of wireless mesh network", International Journal of Engineering and Innovative Technology, 2(6), pp. 338-342, 2012.

[9] Ding, R. Xue, K. Hong, P. and Du, Z, "A novel cluster-based channel assignment scheme for wireless mesh networks", In Consumer Communications and Networking Conference (CCNC). pp.921-925.

[10] Chaudhry, A.U. Hafez, R.H. and Chinneck, J.W, “ On the impact of interference models on channel assignment in multi-radio multi-channel wireless mesh networks", Ad Hoc Networks, 27, pp. 68-80, 2015.

[11] Hoque, M.A. and Hong, X, " Channel assignment algorithms for MRMC wireless mesh networks", International Journal of Wireless \& Mobile Networks, 3(5), 75-94, 2011.

[12] Saini, J.S. and Kumar, R, "Wireless mesh Networks having Topology Control in Multi-Channel, Multi-Radio. International Journal of Electrical, Electronics and Computer Engineering, 2(2), pp.5-17, 2013.

[13] Makram, S.A. and Gunes, M, "Channel assignment for multi-radio wireless mesh networks using clustering", In Telecommunications, ICT, International Conference, pp.1-6. 2008.

[14] Subramanian, A.P. Gupta, H. Das, S.R. and Cao, J, "Minimum interference channel assignment in multi radio wireless mesh networks. IEEE transactions on mobile computing, 7(12), pp.1459-1473, 2008.

[15] Yin, C. Yang, R. Wu, D. and Zhu, W. (2016). Joint multi-channel assignment and routing in wireless mesh network. In Software Engineering, Artificial Intelligence, Networking and Parallel/Distributed Computing (SNPD), International Conference. 26126,2016.

[16] Cooper, I. Allen, S. and Whitaker, R. (2011), "Optimised scheduling for Wireless Mesh Networks using fixed cycle times" In World of Wireless, Mobile and Multimedia Networks (WoWMoM), International Symposium. 1-6, 2011.

[17] Shah, S. Hussain, H. and Shoaib, M, "Minimizing non-coordinated interference in multi-radio multi-channel Wireless Mesh Networks(MRMC WMNs). In Digital Information Management (ICDI M), Eighth International Conference, pp.24-28, 2013. 